\title{
MJMR METHYLENE BLUE INHIBITS THE INTERACTION BETWEEN HEPARAN SULFATE AND SARS-COV-2 SPIKE PROTEIN; A REVIEW OF EVIDENCE FOR A HYPOTHESIS
}

\author{
Neluwa-Liyanage Ruwan Indika ${ }^{1 *}$, Nirmalie Gayathrie Fonseka ${ }^{2}$ \\ ${ }^{1}$ Lecturer, Department of Biochemistry, Faculty of Medical Sciences, University of Sri Jayewardenepura, \\ Nugegoda, Sri Lanka \\ ${ }^{2}$ Medical Officer, National Blood Centre, Colombo 05, Sri Lanka \\ *Corresponding Author's Email: ind.liyanage@sjp.ac.lk
}

\begin{abstract}
The addition of methylene blue to the standard treatment protocol has been shown to improve respiratory rate and oxygen saturation in COVID-19 patients, reducing morbidity and mortality. Evidence to date suggests that methylene blue inhibits protein-protein interactions between SARS-CoV-2 Spike protein and angiotensinconverting enzyme 2, which in turn inhibits the cell entry of SARS-CoV-2. However, the methylene blue dyebinding characteristics of sulfated glycosaminoglycans suggest additional inhibitory effects of the spike proteinheparan sulfate interaction. We hypothesize that the binding of cationic methylene blue neutralizes polyanionic heparan sulfate molecules on the host cell surface. As a consequence, electrostatic interactions between negatively charged heparan sulfate and the positively charged receptor binding domain of SARS-CoV-2 spike protein will be inhibited. Thus, methylene blue will exhibit a "shielding effect" on the heparan sulfate proteoglycans, inhibiting viral attachment to the cell surface. The proposed mechanism corroborates the possible broad-spectrum antiviral activity of methylene blue against multiple human coronaviruses that exploit the electrostatic interactions with sulfated glycosaminoglycans for virus entry. Methylene blue would exhibit the same anti-adhesive activity at the blood-brain-barrier and olfactory neuroepithelium, corroborating potential benefits in ameliorating post-COVID-19 neurological impairment. However, as cationic dyes can bind to both free glycosaminoglycans in circulation as well as proteoglycans attached to the cell surface, co-administration of intravenous heparin could possibly antagonize the proposed antiviral activity. This critical review focuses on empirical evidence to support the hypothesized heparan sulfate-dependent antiviral activity of MB.
\end{abstract}

\section{Keywords: Methylene Blue; SARS-Cov-2; COVID-19, Heparan Sulfate; Proteoglycans; Antiviral}

\section{INTRODUCTION}

Methylene blue (MB) is a medication used for both diagnostic and therapeutic purposes in different areas of the medical field. MB appears to demonstrate multiple biochemical mechanisms to bring about desired effects depending on its use. For instance, intravenous administration of MB for emergency treatment of methemoglobinemia is a Food and Drug Administration (FDA)-approved therapeutic indication where it acts by reducing the oxidized form of $\mathrm{Fe} 3+$ in methemoglobin to $\mathrm{Fe} 2+$ (Bistas \& Sanghavi, 2020). Even though the remaining therapeutic indications, such as vasoplegic syndrome, septic shock, Plasmodium falciparum malaria, and photodynamic cancer therapy, are not FDA-approved, diverse biochemical mechanisms of the drug are of great interest (Ginimuge \& Jyothi, 2010).

Preliminary results of a phase-I clinical trial showed coadministration of $\mathrm{MB}$, vitamin $\mathrm{C}$, and $\mathrm{N}$-acetyl cysteine decreased inflammatory markers and resulted in overall clinical improvement in COVID-9 patients (HamidiAlamdari et al., 2020). Moreover, a randomized, controlled, open-label clinical trial conducted by the same research group showed that the addition of MB to the treatment protocol significantly improved respiratory rate and oxygen saturation in COVID-19 patients, which in turn decreased hospital stay and mortality. Furthermore, a number of therapeutic roles of MB in COVID-19 have been postulated (HamidiAlamdari et al., 2021).

In the light of evidence coming from initial clinical trials, MB has been a point of discussion in several hypotheses and literature reviews as a multifunctional agent. Among the pathogenic mechanisms of COVID19 that can be targeted by MB are inhibition of SARSCoV-2-host cell interaction, phagosome maturation, translation of viral RNA, viral replication, hypoxic 
damage, hyper-inflammatory reaction, etc (Pardo Andreu, 2021). Moreover, MB has other potential applications in the COVID-19 pandemic, including decontamination of personal protective equipment (Lendvay et al., 2021), and pathogen reduction of COVID-19 convalescent plasma (Kostin et al., 2021). Additionally, some studies suggest the prospective use of photodynamic therapy as an effective method for the prevention and treatment of COVID-19 (Svyatchenko et al., 2021). However, MBhas also demonstrated in vitro antiviral activity in non-photodynamic assays (Gendrot et al., 2020). This critical review focuses on empirical evidence to support the heparan sulfatedependent antiviral activity of MB.

\section{METHODOLOGY}

The present hypothesis proposes that aggregation of methylene blue on heparan sulfateproteoglycans on the host cell surface will inhibit the binding of SARS-CoV2 spike proteins. A systematic approach was applied to substantiate the hypothesis whichwas conceptualized based on three complementary empirical findings.

1. Methylene Blue inhibits cellular entry of SARSCoV-2.

2. Methylene blue binds to and forms complexes with polyanionic glycosaminoglycans (GAGs).

3. Adhesion of SARS-CoV-2 through spike proteinheparan sulfate interactions facilitates the cellular entry of SARS-CoV-2.

Online electronic bibliographic databases such as PubMed, Scopus, and Google Scholar were explored using specific search strings limiting the search to the title, abstract, and keywords. The search terms used were "methylene blue", "antiviral", "heparan", "glycosaminoglycan", "SARS-CoV-2", "coronavirus", and "COVID-19". Search results were pooled and duplicates were removed. Irrelevant papers are eliminated by examining abstracts while the relevant scholarly literature is perused and woven into the hypothesis. Additional sources were also retrieved from the reference lists of eligible papers and review articles.

The subsequent sections of the present paper are stratified in terms of the three complementary empirical findings that led to the hypothesis.

\section{RESULTS}
Methylene Blue Inhibits Cellular Entry of SARS- CoV-2

MB has been found to exhibitpotent antiviral activity against SARS-CoV-2 in the absence of UV- activation in vitro (Cagno et al., 2021). It has been demonstrated thatthe drug inhibits the entry of a SARS-CoV-2 spike bearing pseudovirus into angiotensin-converting enzyme 2 (ACE2)-expressing cells (Bojadzic et al, 2020). Subsequently, a study conducted by the same research group suggested that MB inhibits proteinprotein interactions between SARS-CoV-2 Spike protein and ACE2 (Bojadzic et al., 2021).On the other hand, another study showed thatthe addition of MB to the cell cultures inhibits the replication of SARS-CoV2 at both "entry" and "post-entry" stages supporting the multimodal nature of its action (Gendrot et al., 2020; Gendrot et al., 2021). Therefore, the drug may exhibit multiple mechanisms of action at both entry and postentry stages of the infection, in addition to inhibition of SARS-CoV-2 spike protein-ACE2 interaction. The inhibition of SARS-CoV-2-heparan sulfate interaction has not been proposed previously as a potential mechanism of action.

\section{Interactions between heparan sulfate and SARS- CoV-2 spike protein}

SARS-CoV-2 uses ACE2 as the primary entry receptor (Hassanzadeh et al., 2020). Nevertheless, directed expression or selective cleavage of ACE2 had no measurable effect on viral adhesion (Milewska et al., 2014). Conversely, ablation of genes involved in heparan sulfate biosynthesis or incubating cells with a heparan sulfatemimetic inhibited Spike-mediated viral entry (Zhang et al., 2020). Therefore, these findings suggest that SARS-CoV-2 initially binds to cell surface heparan sulfateincreasing virus density at the cell surface and facilitating the interaction between SARSCoV-2 and ACE2 for virus entry (Reis et al., 2021), as illustrated in Fig. 1A. Moreover, microarray binding experiments have suggested that ACE2 and heparan sulfate can simultaneously engage with the receptorbinding domain (RBD) of SARS-CoV-2 spike protein, and probably no dissociation between heparan sulfateand RBD is required for binding to ACE2 (Liu et al., 2021). Furthermore, studies have revealed significant colocalization between SARS-CoV-2 and syndecan (a proteoglycan), suggesting a jointly shared internalization pathway.Among syndecans, the lung abundant syndecan- 4 was found to be the most efficient in mediating SARS-CoV-2 uptake (Hudák et al., 2021). In line with the aforementioned findings, heparan sulfatehas been recognized as an important attachment factor for SARS-CoV-2 infection in human lung 
epithelial cells (Chu et al., 2021). Importantly, SARSCoV-2 spike glycoprotein has shown a significantly higher affinity to human lung heparan sulfatethan bat lung heparan sulfate (Yan et al., 2021). The higher infectivity of the SARS-CoV-2 can be partly attributed to the highaffinity of the spike glycoprotein to heparan sulfate (Yue et al., 2021).

Molecular docking studies suggest that SARS-CoV-2 spike protein interacts with heparan sulfate and ACE2 through multiple binding sites on the $\mathrm{RBD}$ (Clausen et al., 2020; Schuurs et al., 2021). Furthermore, the SARS-CoV-2 spike protein is slightly more positively charged than that of SARS-CoV since it contains four more positively charged residues and five less negatively charged residues. Therefore, it has been suggested that SARS-CoV-2 spike protein exhibits an increased affinity to bind with negatively charged adhesion molecules (Hassanzadeh et al., 2020). Heparan sulfate, which belongs to the group of sulfated GAGs, are polyanions with high negative charge density (Soares da Costa et al., 2017). Hence, heparan sulfatecan form strong electrostatic interactions with RBD of SARS-CoV-2 spike protein. Therefore, drugs targeting the interaction between heparan sulfateand SARS-CoV-2-related proteins have been proposed as an important strategy for developing novel therapeutics for the ongoing COVID-19 pandemic (Yu et al., 2021; Zhang et al., 2020). Highly sulfated GAGs such as heparin and tri-sulfated heparan sulfate have higher charge density and binds SARS-CoV-2 spikeprotein with a higher affinity. The position of sulfation within heparin was also found to be important for its successfulbinding to SARS-CoV-2 spike protein (Hao et al., 2021; Kim et al., 2020). Therefore, nonanticoagulant derivatives of heparin, heparan sulfate mimetics, and other sulfated and sulfonated moleculeshave been suggested to have a therapeutic potential against SARS-CoV-2 infection ascompetitive inhibitors (Gasbarri et al., 2020; Jin et al., 2020; Kwon et al., 2020; Mycroft-West et al., 2020; Tandon et al., 2021; Wells et al., 2021). Importantly, inhibition of viral cellular entry is one of the potential nonanticoagulant rolesin heparin therapy of COVID-19 patients (Buijsers et al., 2020).

Intriguingly, attachment of the virus to heparan sulfateproteoglycans on host cells is a common step in the lifecycle of multiple viruses, including herpesviruses, human immunodeficiency virus, dengue virus, human papillomavirus, as well as coronaviruses (Koganti et al., 2021). Therefore, discovering an effective drug targeting heparan sulfate will open up a new paradigm in antiviral therapeutics In line with this prospect, a study revealed that bovine lactoferrin binds to heparan sulfateproteoglycans, thereby blocking viral attachment to the host cell. The same study showed that lactoferrin has broad-spectrum antiviral activity against multiple coronaviruses including SARS-CoV-2 (Hu et al., 2021). Interestingly, lactoferrin did not have an inhibitory effect on thespike protein-ACE2 interaction (Lang et al., 2011). Therefore, it could be hypothesized that heparan sulfate-binding drugs that inhibit spike protein-heparan sulfate interaction will show a broad-spectrum antiviral activity. Several drugs and heparin/heparan sulfate binding peptides that inhibit viral entry by targeting heparan sulfate have been proposed and studied (Suryawanshi et al., 2021; Tavassoly et al., 2020; Zhang et al., 2020). Human monoclonal antibodies targeting the SARS-CoV-2 spike protein have already entered clinical trials while other viral attachment sites such as heparan sulfateproteoglycans have been suggested as potential targets (Sun and Ho, 2020). Despite this interest, to the best of our knowledge, no research or hypothesis has focused on the potential heparan sulfate-dependent anti-adhesive activity of MB against SARS-CoV-2.

\section{Interaction between heparan sulfate and cationic dyes}

Metachromatic dyes such as MB, dimethylmethylene blue (DMMB), and toluidine blue are positively charged and react with negatively charged polyanions in aqueous solutions. The binding of the dye with the polyanions neutralizes the positively charged dye. Consequently, the non-polar aromatic ring of the dye binds with the adjacent dye by Van der Waals forces releasing the water bound tothe dye molecules and resulting in dye-to-dye aggregation. The overall binding of the dye becomes stronger due to the Van der Waals forces and the absorbance spectrum of the dye shifts to a shorter wavelength(Dey, 2018; Jakubowska et al., 2014; Jiao and Liu, 1999). This principle is used to detect GAGs in biological samples (Stone et al., 1994; Templeton, 1988; Zhang et al., 2004).MB can bind to both free and protein-bound forms of heparan sulfate, given that the size is over fourdisaccharide units (Németh-Csóka et al., 1975). Therefore heparan sulfate proteoglycans on the host cell surface will possibly exhibit the aforementioned dye-binding characteristics during MB therapy.

\section{DISCUSSION}

In the light of empirical evidence, it can be conceivably hypothesized that cationic dyes such as MB can form 
aggregates on heparan sulfatemolecules covalently linked to core proteins (proteoglycans) and interfere with electrostatic interactions between negatively charged heparan sulfateand positively charged patch in RBD. Thus, cationic dye molecules will exhibit a "shielding effect" on the heparan sulfateproteoglycans as illustrated in Fig. 1B. This will inhibit the heparan sulfate-mediated viral attachment and decrease the local concentration of pathogens on the cell surface. Thus, itrenders the interaction of SARS-CoV-2 with the entry receptors less efficient. Therefore, cationic dyes such as MB may exhibit anti-adhesion properties in addition to direct inhibition of spike protein-ACE2 interaction.

Considering the non-specific nature of the proposed mechanism of action, it can be further hypothesized that cationic dyes could exertbroad-spectrum antiviral activity against multiple human coronaviruses that exploit the electrostatic interactions with sulfated GAGs for virus entry. However, cationic dyes can bind to both free GAGs in circulation as well as proteoglycans attached to the cell surface. On the other hand, the charge density of heparin is higher than that of heparan sulfate. Therefore, co-administration of intravenous heparin can possibly antagonize the proposed antiviral activity of MB.

Transneuronal spread through the olfactory nerves and hematogenousroute after breaching the blood-brain barrier have been proposed as possible routes for SARS-CoV-2 brain entry (Kumar et al., 2020). Expression of the viral binding receptors in non-neural cells of the olfactory epithelium and myelin-forming cells are implicated in facilitating the invasion of olfactory nerve. Interestingly, the olfactory neuroepithelium has been shown to express heparan sulfateimportant and strong binding sites for herpes virus entry (Milho et al., 2012). Moreover, brain microvascular endothelial cells express high levels of heparan sulfateand chondroitin sulfates which act as binding sites for brain-tropic viruses via electrostatic interactions between basic residues in viral glycoproteins and negatively charged sulfated GAGs (Bobardt et al., 2004). This evidence suggests that SARS-CoV-2 may exploit its electrostatic interactions with sulfated GAGs to enter and migrate through the blood-brain barrier and olfactory epithelium to invade the brain. Therefore, cationic dyes would possibly exhibit the same anti-adhesive activity at the bloodbrain-barrier and olfactory neuroepithelium corroborating potential benefits in ameliorating postCOVID-19 neurological impairment. Therefore, this may add an incremental value to MB therapy as a neuroprotectiveagentin COVID-19.

$M B$ is a safe medication when used within its therapeutic range $<2 \mathrm{mg} / \mathrm{kg}$. It is water-soluble and can be administered by oral, intranasal, inhalational as well as intravenous routes. The drug is inexpensive and widely available across the world. Moreover, compared to protein therapeutics such as antibodies, MB therapy has a relatively lower propensity for unwanted immunogenicity and hypersensitivity. The other advantages over conventional antivirals include inhibition of excessive production of reactive species and cytokines, and amelioration of post-COVID-19 blues (Magoon et al., 2021; Scigliano \& Scigliano, 2021). Further, it has the possible advantage of having antibacterial effects against secondary bacterial infections commonly encountered among COVID-19 patients. Nevertheless, MB is contraindicated in pregnancy and glucose-6-phosphate dehydrogenase deficiency (Bistas\&Sanghavi, 2020). MB can also give rise to falsely lower estimates of oxygen saturation in COVID-19 patients due to its interference with the pulse oximeter's light emission (Ginimuge\&Jyothi, 2010).

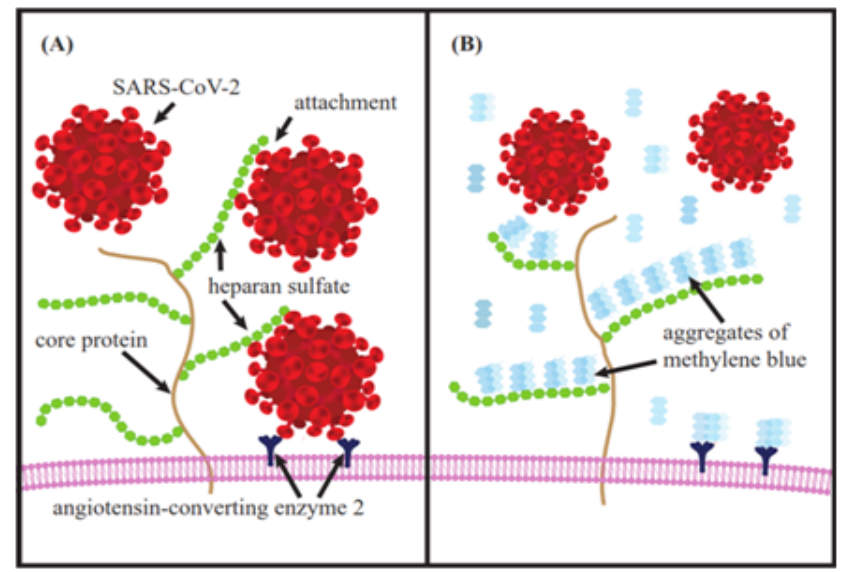

Figure 1; (A) SARS-CoV-2 initially binds to cell surface heparan sulfate and facilitates the interaction between SARS-CoV-2 and angiotensin-converting enzyme 2. (B) Methylene blue forms aggregates on heparansulfate molecules and blocks viral attachment.

\section{CONCLUSIONS}

A plethora of evidence corroborates the proposed hypothesis in which MB binds to heparan sulfate and inhibits SARS-CoV-2-heparan sulfate interactions. We believe that the present hypothesis will form a base for future studies focusing on the effect of cationic 
molecules on heparan sulfate-mediated viral adhesion/attachment. We suggest that the hypothesized effect of $\mathrm{MB}$ can be tested by conducting flow cytometry-based and confocal microscopy-based virus adhesion experiments on cells with heparan sulfateproteoglycan expression. Due to the prevailing COVID-19 pandemic, the attempts to repurpose the existing drugs for the treatment of COVID-19 are pertinent. MB appears to be a good candidate for drug repurposing with both preventive and curative prospects, considering the evidence supporting antiviral and other beneficial actions. More broadly, future research should also examine the approaches for novel drug discovery targeting heparan sulfatemediated virus adhesion.

\section{Authors' Contributions}

NLR. Indika conceived the hypothesis, created the graphical illustrations, reviewed the literature and drafted the manuscript. NG. Fonseka participated in developing concept outline, interpretation of literature and revising the manuscript critically for important intellectual content. All the authors have read and approved the final manuscript.

\section{Conflict of interest}

The authors declare that they have no competing interests in writing this article.

\section{ACKNOWLEDGEMENT}

The authors would like to thank Dr.Udara D. Senarathnewho moderated this paper and in that line improved the manuscript significantly.

\section{Funding}

This work did not receive any specific grant from funding agencies in the public, commercial, or not-forprofit sectors.

\section{REFERENCES}

Buzon, M. J., Seiss, K., Weiss, R., Brass, A. L., Rosenberg, E. S., Pereyra, F., ... \& Lichterfeld, M. (2011). Inhibition of HIV-1 integration in ex vivoinfected CD4 T cells from elite controllers. Journal of virology, 85(18), 9646-9650.

Bojadzic, D., Alcazar, O., \& Buchwald, P. (2021). Methylene blue inhibits the SARS-CoV-2 spike-ACE2 protein-protein interaction-a mechanism that can contribute to its antiviral activity against COVID-19. Frontiers in

\section{Pharmacology, 2255.}

Bojadzic, D., Alcazar, O., Chen, J., Chuang, S. T., Condor Capcha, J. M., Shehadeh, L. A., \& Buchwald, P. (2021). Small-Molecule Inhibitors of the Coronavirus Spike: ACE2 Protein-Protein Interaction as Blockers of Viral Attachment and Entry for SARS-CoV-2. ACS Infectious Diseases.

Buijsers, B., Yanginlar, C., Maciej-Hulme, M. L., de Mast, Q., \& van der Vlag, J. (2020). Beneficial nonanticoagulant mechanisms underlying heparin treatment of COVID-19 patients. EBioMedicine, 59,102969 .

Cagno, Valeria, Chiara Medaglia, Andreas Cerny, Thomas Cerny, Caroline Tapparel, and Erich Cerny. "Methylene blue has a potent antiviral activity against SARS-CoV-2 in the absence of UVactivation in vitro." bioRxiv (2020).

Chu, H., Hu, B., Huang, X., Chai, Y., Zhou, D., Wang, Y., ... \& Yuen, K. Y. (2021). Host and viral determinants for efficient SARS-CoV-2 infection of the human lung. Nature communications, 12(1), 1-15.

Clausen, T. M., Sandoval, D. R., Spliid, C. B., Pihl, J., Perrett, H. R., Painter, C. D., ... \& Esko, J. D. (2020). SARS-CoV-2 Infection Depends on Cellular Heparan Sulfate and ACE2. Cell. https. doi. org/10.1016/j. cell, 33 .

Dey, P. (2018). Staining Principle and general procedure of staining of the tissue. In Basic and Advanced Laboratory Techniques in Histopathology and Cytology (pp. 57-67). Springer, Singapore.

Gasbarri, M., V'kovski, P., Torriani, G., Thiel, V., Stellacci, F., Tapparel, C., \& Cagno, V. (2020). Sars-cov-2 inhibition by sulfonated compounds. Microorganisms, 8(12), 1894.

Gendrot, M., Andreani, J., Duflot, I., Boxberger, M., Le Bideau, M., Mosnier, J., ... \& Pradines, B. (2020). Methylene blue inhibits replication of SARS-CoV2 in vitro. International Journal of Antimicrobial Agents, 56(6), 106202.

Gendrot, M., Jardot, P., Delandre, O., Boxberger, M., Andreani, J., Duflot, I., ... \& Pradines, B. (2021). In Vitro Evaluation of the Antiviral Activity of Methylene Blue Alone or in Combination against SARS-CoV-2. Journal of Clinical Medicine, 
10(14), 3007.

Ginimuge, P. R., \& Jyothi, S. (2010). Methylene blue: revisited. Journal of anaesthesiology, clinical pharmacology, 26(4), 517.

Hamidi-Alamdari, D., Hafizi-Lotfabadi, S., BagheriMoghaddam, A., Safari, H., Mozdourian, M., Javidarabshahi, Z., ... \& Koliakos, G. (2021). Methylene blue for treatment of hospitalized COVID-19 patients: A randomized, controlled, open-label clinical trial, phase 2. Revista de investigación clínica, 73(3), 190-198.

Hao, W., Ma, B., Li, Z., Wang, X., Gao, X., Li, Y., ... \& Tan, Z. (2021). Binding of the SARS-CoV-2 spike protein to glycans. Science Bulletin, 66(12), 12051214.

Hassanzadeh, K., Perez Pena, H., Dragotto, J., Buccarello, L., Iorio, F., Pieraccini, S., ... \& Feligioni, M. (2020). Considerations around the SARS-CoV-2 Spike Protein with particular attention to COVID-19 brain infection and neurological symptoms. ACS chemical neuroscience, 11(15), 2361-2369.

Hu, Y., Meng, X., Zhang, F., Xiang, Y., \& Wang, J. (2021). The in vitro antiviral activity of lactoferrin against common human coronaviruses and SARS$\mathrm{CoV}-2$ is mediated by targeting the heparan sulfate co-receptor. Emerging microbes \& infections, 10(1), 317-330.

Hudák, A., Letoha, A., Szilák, L., \& Letoha, T. (2021). Contribution of syndecans to the cellular entry of SARS-CoV-2. International journal of molecular sciences, 22(10), 5336.

Hudák, A., Letoha, A., Szilák, L., \& Letoha, T. (2021). Contribution of syndecans to the cellular entry of SARS-CoV-2. International journal of molecular sciences, 22(10), 5336.

Indika, N. L. R., \& Fonseka, N. G. (2021). METHYLENE BLUE INHIBITS THE INTERACTION BETWEEN HEPARAN SULFATE AND SARS-COV-2 SPIKE PROTEIN; A REVIEW OF EVIDENCE FOR A HYPOTHESIS. Malaysian Journal of Medical Research (MJMR), 5(4).

Jiao, Q., \& Liu, Q. (1999). Characterization of the interaction between methylene blue and glycosaminoglycans. Spectrochimica Acta Part A: Molecular and Biomolecular Spectroscopy, 55(78), 1667-1673.

Jin, W., Zhang, W., Mitra, D., McCandless, M. G., Sharma, P., Tandon, R., ... \& Linhardt, R. J. (2020). The structure-activity relationship of the interactions of SARS-CoV-2 spike glycoproteins with glucuronomannan and sulfated galactofucan from Saccharina japonica. International journal of biological macromolecules, 163, 1649-1658.

Kim, S. Y., Jin, W., Sood, A., Montgomery, D. W., Grant, O. C., Fuster, M. M., ... \& Linhardt, R. J. (2020). Characterization of heparin and severe acute respiratory syndrome-related coronavirus 2 (SARS-CoV-2) spike glycoprotein binding interactions. Antiviral research, 181, 104873.

Koganti, R., Memon, A., \& Shukla, D. (2021, April). Emerging roles of heparan sulfate proteoglycans in viral pathogenesis. In Seminars in Thrombosis and Hemostasis (Vol. 47, No. 03, pp. 283-294). Thieme Medical Publishers, Inc..

Kostin, A. I., Lundgren, M. N., Bulanov, A. Y., Ladygina, E. A., Chirkova, K. S., Gintsburg, A. L., ... \& Petrikov, S. S. (2021). Impact of pathogen reduction methods on immunological properties of the COVID-19 convalescent plasma. Vox Sanguinis.

Kumar, A., Pareek, V., Prasoon, P., Faiq, M. A., Kumar, P., Kumari, C., \& Narayan, R. K. (2020). Possible routes of SARS-CoV-2 invasion in brain: in context of neurological symptoms in COVID-19 patients. Journal of neuroscience research, 98(12), 23762383.

Kwon, P. S., Oh, H., Kwon, S. J., Jin, W., Zhang, F., Fraser, K., ... \& Dordick, J. S. (2020). Sulfated polysaccharides effectively inhibit SARS-CoV-2 in vitro. Cell discovery, 6(1), 1-4.

Lang, J., Yang, N., Deng, J., Liu, K., Yang, P., Zhang, G., \& Jiang, C. (2011). Inhibition of SARS pseudovirus cell entry by lactoferrin binding to heparan sulfate proteoglycans. PloS one, 6(8), e23710.

Lendvay, T. S., Chen, J., Harcourt, B. H., Scholte, F. E., Lin, Y. L., Kilinc-Balci, F. S., ... \& Chu, M. C. (2020). Addressing Personal Protective Equipment 
(PPE) Decontamination: Methylene Blue and Light Inactivates SARS-CoV-2 on N95 Respirators and Medical Masks with Maintenance of Integrity and Fit. Infection Control \& Hospital Epidemiology, 183.

Liu, L., Chopra, P., Li, X., Bouwman, K. M., Tompkins, S. M., Wolfert, M. A., ... \& Boons, G. J. (2021). Heparan sulfate proteoglycans as attachment factor for SARS-CoV-2. ACS Central Science.

Magoon, R., Bansal, N., Singh, A., \& Kashav, R. (2021). Methylene blue: Subduing the post COVID-19 blues!. Medical Hypotheses.

Milewska, A., Zarebski, M., Nowak, P., Stozek, K., Potempa, J., \& Pyrc, K. (2014). Human coronavirus NL63 utilizes heparan sulfate proteoglycans for attachment to target cells. Journal of virology, $88(22), 13221-13230$.

Milho, R., Frederico, B., Efstathiou, S., \& Stevenson, P. G. (2012). A heparan-dependent herpesvirus targets the olfactory neuroepithelium for host entry. PLoS pathogens, 8(11), e1002986

Mycroft-West, C. J., Su, D., Pagani, I., Rudd, T. R., Elli, S., Guimond, S. E., ... \& Skidmore, M. A. (2020). Heparin inhibits cellular invasion by SARS-CoV-2: structural dependence of the interaction of the surface protein (spike) $\mathrm{S} 1$ receptor binding domain with heparin. BioRxiv.

Németh-Csóka, M., Kajtár, J., \& Kajtár, M. (1975). Biological significance of helical conformation of acid polysaccharides. Connective tissue research, 3(4), 207-211.

Andreu, G. L. P. (2021). The rationale for methylene blue utility against SARS-CoV-2 infection complications. Journal of Pharmacy \& Pharmacognosy Research, 9(3), 379-396.

Reis, C. A., Tauber, R., \& Blanchard, V. (2021). Glycosylation is a key in SARS-CoV-2 infection. Journal of Molecular Medicine, 1-9.

Schuurs, Z. P., Hammond, E., Elli, S., Rudd, T. R., Mycroft-West, C. J., Lima, M. A., ... \& Gandhi, N. S. ( 2021$)$. Evidence of a putative glycosaminoglycan binding site on the glycosylated SARS-CoV-2 spike protein Nterminal domain. Computational and Structural
Biotechnology Journal, 19, 2806-2818.

Scigliano, G., \& Scigliano, G. A. (2021). Methylene blue in covid-19. Medical Hypotheses, 146, 110455.

Soares da Costa, D., Reis, R. L., \& Pashkuleva, I. (2017). Sulfation of glycosaminoglycans and its implications in human health and disorders. Annual review of biomedical engineering, 19, 1-26.

Stone, J. E., Akhtar, N., Botchway, S., \& Pennock, C. A. (1994). Interaction of 1, 9-dimethylmethylene blue with glycosaminoglycans. Annals of clinical biochemistry, 31(2), 147-152.

Sun, Y., \& Ho, M. (2020). Emerging antibody-based therapeutics against SARS-CoV-2 during the global pandemic. Antibody therapeutics, 3(4), 246256.

Suryawanshi, R. K., Patil, C. D., Koganti, R., Singh, S. K., Ames, J. M., \& Shukla, D. (2021). Heparan Sulfate Binding Cationic Peptides Restrict SARSCoV-2 Entry. Pathogens, 10(7), 803.

Svyatchenko, V. A., Nikonov, S. D., Mayorov, A. P., Gelfond, M. L., \& Loktev, V. B. (2021). Antiviral photodynamic therapy: Inactivation and inhibition of SARS-CoV-2 in vitro using methylene blue and Radachlorin. Photodiagnosis and Photodynamic Therapy, 33, 102112.

Tandon, R., Sharp, J. S., Zhang, F., Pomin, V. H., Ashpole, N. M., Mitra, D., ... \& Linhardt, R. J. (2020). Effective inhibition of SARS-CoV-2 entry by heparin and enoxaparin derivatives. Journal of virology, 95(3), e01987-20.

Tavassoly, O., Safavi, F., \& Tavassoly, I. (2020). Heparin-binding Peptides as Novel Therapies to Stop SARS-CoV-2 Cellular Entry and Infection. Molecular Pharmacology, 98(5), 612-619.

Templeton, D. M. (1988). The basis and applicability of the dimethylmethylene blue binding assay for sulfated glycosaminoglycans. Connective Tissue Research, 17(1), 23-32.

Wells, L., Vierra, C., Hardman, J., Han, Y., Dimas, D., Gwarada-Phillips, L. N., ... \& McReynolds, K. D. (2021). Sulfoglycodendrimer Therapeutics for HIV-1 and SARS-CoV-2. Advanced therapeutics, 4(4), 2000210. 
Yan, L., Song, Y., Xia, K., He, P., Zhang, F., Chen, S., ... \& Linhardt, R. J. (2021). Heparan sulfates from bat and human lung and their binding to the spike protein of SARS-CoV-2 virus. Carbohydrate Polymers, 260, 117797.

Yu, M., Zhang, T., Zhang, W., Sun, Q., Li, H., \& Li, J. P. (2020). Elucidating the interactions between heparin/heparan sulfate and SARS-CoV-2-related proteins-An important strategy for developing novel therapeutics for the COVID-19 pandemic. Frontiers in Molecular Biosciences, 7.

Yue, J., Jin, W., Yang, H., Faulkner, J., Song, X., Qiu, H., ... \& Wang, L. (2021). Heparan sulfate facilitates spike protein-mediated SARS-CoV-2 host cell invasion and contributes to increased infection of SARS-CoV-2 G614 mutant and in lung cancer. Frontiers in Molecular Biosciences, 8.

Zhang, L., Li, N., \& Zhao, F. (2004). Spectroscopic study on the interaction between methylene blue and chondroitin 4-sulfate and its analytical application. Analytical sciences, 20(3), 445-450.

Zhang, Q., Chen, C.Z., Swaroop, M., Xu, M., Wang, L., Lee, J., . . Y Ye, Y. (2020). Heparan sulfate assists SARS-CoV-2 in cell entry and can be targeted by approved drugs in vitro. Cell Discov, 6(1), 80. 